\title{
14.
}

\section{Effects of cortisol implant on innate and acquired immunity in sea bass Dicentrarchus labrax L}

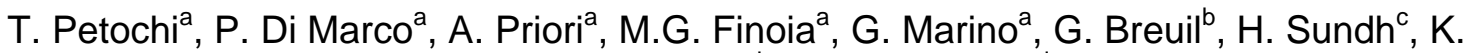 \\ Sundell ${ }^{\mathrm{c}}$, E. Caccia ${ }^{\mathrm{d}}$ and N. Romano ${ }^{\mathrm{d}}$
}

\footnotetext{
a ICRAM Central Institute for Marine Research, Rome, Italy

${ }^{\mathrm{b}}$ IFREMER, Palavàs-les-Flots, France

${ }^{\mathrm{C}}$ Department of Zoology, University of Göteborg, Sweden

d Department of Environmental Sciences, University of Tuscia, Italy
}

\begin{abstract}
This study investigates the effects of high plasma cortisol levels induced by slow release intraperitoneal cortisol implantation on the innate and acquired immune system of cultured sea bass. Sub-adult fish were kept in recycling tanks $(1 \mathrm{~m} 3)$ at $20.0 \pm 0.5{ }^{\circ} \mathrm{C}$ and exposed for 5 weeks to three different treatments in duplicate: non implanted fish (control), vehicle implanted fish and fish implanted with $75 \mu \mathrm{g} / \mathrm{g}$ body weight of cortisol (IP-75). Sea bass were sampled 1,3 and 5 weeks after implantation and analysed for serum cortisol, complement (ACH50) and lysozyme (LYS) activities. After 5 weeks the percentage of B and T lymphocytes in head kidney (HK) and peripheral blood (PBL) was also measured. Plasma cortisol levels increased dramatically after 1 week from implantation in IP75 and decreased after 3 weeks, although plasma levels were still higher than in other groups. No differences among groups were detected after 5 weeks. LYS decreased significantly in IP-75 after 1 and 3 weeks and ACH50 after 3 weeks. When normal cortisol levels were restored in IP-75 (5 weeks), no differences in innate immune parameters were observed among groups. Conversely, after 5 weeks the percentage of B and T lymphocytes in PBL were significantly lower in IP-75 compared to other groups. The percentage of HK lymphocytes did not differ among groups. Cortisol is the primary stress hormone released following activation of HPI-axis in teleosts. High plasma cortisol is reported to affect the immune system in different fish species. Our results demonstrated that high circulating cortisol levels affect both innate and acquired immune response in sea bass and may increase disease susceptibility.
\end{abstract}

